\title{
A novel method to address the association between received dose intensity and survival outcome: benefits of approaching treatment intensification at a more individualised level in a trial of the European Osteosarcoma Intergroup
}

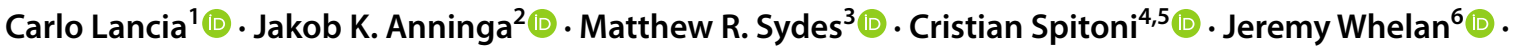 \\ Pancras C. W. Hogendoorn ${ }^{7}$ (D) Hans Gelderblom ${ }^{8}$ () $\cdot$ Marta Fiocco $^{1,9}$
}

Received: 8 November 2018 / Accepted: 8 February 2019 / Published online: 16 March 2019

(c) The Author(s) 2019

\begin{abstract}
Purpose There is lack of consensus on the prognostic value of received high dose intensity in osteosarcoma survivorship. Many studies have not shown a clear survival benefit when dose intensity is increased. The aim of this study is to go beyond chemotherapy intensification by arm-wide escalation of intended dose and/or compression of treatment schedule, while conversely addressing the relationship between treatment intensity and survival at the patient level. The study focusses on the difference in outcome results, based on a novel, progressively more individualised approach to dose intensity.

Methods A retrospective analysis of data from MRC BO06/EORTC 80931 randomised controlled trial for treatment of osteosarcoma was conducted. Three types of post hoc patient groups are formed using the intended regimen: the individually achieved cumulative dose and time on treatment, and the increase of individual cumulative dose over time. Event-free survival is investigated and compared in these three stratifications.

Results The strata of intended regimen and achieved treatment yields equivalent results. Received cumulative dose over time produces groups with evident different survivorship characteristics. In particular, it highlights a group of patients with an estimated 3-year event-free survival much larger (more than 10\%) than other patient groups. This group mostly contains patients randomised to an intensified regimen. In addition, adverse events reported by that group show the presence of increased preoperative myelotoxicity.

Conclusions The manuscript shows the benefits of analyzing studies by using longitudinal data, e.g. recorded per cycle. This has impact on the drafting of future trials by showing why such a level of detail is needed for both treatment and adverse event data. The novel method proposed, based on cumulative dose received over time, shows that longitudinal treatment data might be used to link survival outcome with drug metabolism. This is particularly valuable when pharmacogenetics data for metabolism of cytotoxic agents are not collected.
\end{abstract}

Trial registration ISRCTN86294690.

Keywords Bone tumour $\cdot$ Sarcoma $\cdot$ Chemotherapy $\cdot$ Personalised medicine

Abbreviations
$\begin{array}{ll}\text { RDI } & \text { Received dose intensity } \\ \text { tRDI } & \text { Target RDI } \\ \text { aRDI } & \text { Achieved RDI }\end{array}$

Carlo Lancia

c.lancia@math.leidenuniv.nl

Cristian Spitoni

c.spitoni@uu.nl

Extended author information available on the last page of the article
rRDI Regulated RDI

EFS Event-free survival

\section{Introduction}

Received dose intensity (RDI) was first described by Hryniuk as the given dose (in $\mathrm{mg} / \mathrm{m}^{2}$ ) during a certain time period [1]. This definition applies to either single cytostatic agents or drug combinations [2]. The prognostic value of high RDI in cancer survivorship has been discussed in 
several studies [3-8] and for many cancer types; however, this debate has never reached consensus $[9,10]$.

In osteosarcoma, RDI increase can be achieved by schedule compression supported by granulocyte colony-stimulating factor, e.g. by comparing 3-weekly doxorubicin plus cisplatin with a 2-weekly administration of the same agents at the same dosage [11]. This led to a 31\% RDI increase and a significantly higher proportion of good histologic response in the intensified arm compared to the uncompressed control arm. Yet unexpectedly, an increase of RDI was not associated with a better survival, leading to the conclusion that histologic response was not a good surrogate marker tout court for survival outcome in osteosarcoma, because its prognostic value is limited to homogeneous groups of patients.

Nevertheless, treatment decisions have been often based on histologic response [12]. However, the recently closed EURAMOS-1 study showed no evidence that regimen intensification yields better survival outcome for poor histologic response [13]. Other studies have not shown a clear survival benefit when increasing dose intensity in osteosarcoma [14], perhaps indicating that the effect of treatment intensification should not be addressed by cohort comparison.

Treatment-induced toxicity has been shown to vary among different groups of age and gender [15]. Recently, it has been shown that variant expression of genes, involved in the metabolism of methotrexate, cisplatin and doxorubicin is related to progression-free survival in osteosarcoma [16, 17]. This finding suggests the presence of inter-patient heterogeneity for metabolism of cytotoxic agents. Finally, there is evidence that lymphopenia is an independent prognostic factor for overall and progression-free survival in several cancers [18].

Besides pharmacogenetics, there are a number of other factors driving differences in RDI, many of which vary at the patient level. The aim of this study is to gain insight into the treatment effects in osteosarcoma. Thus, we analyse the difference in outcome results that is observed by using a progressively more individualised approach to dose intensity.

\section{Patients and methods}

\section{Patients}

Data for this study were collected from the MRC BO06/ EORTC 80931 (ISRCTN86294690) randomised controlled trial for patients with newly diagnosed, resectable high-grade osteosarcoma. The trial compared pathological response and survival outcome of the control regimen (RegC), six courses of 3-weekly doxorubicin (DOX, $75 \mathrm{mg} / \mathrm{m}^{2}$ ) and cisplatin (CDDP, $100 \mathrm{mg} / \mathrm{m}^{2}$ ), versus a dose-intense regimen (Reg-DI), same courses administered 2-weekly and supported by the hematopoietic growth factor G-CSF.
Chemotherapy was given perioperatively. Surgical resection of primary osteosarcoma was scheduled at week 6 since the start of treatment, i.e. after $2 \times \mathrm{DOX}+\mathrm{CDDP}$ for Reg-C and after $3 \times$ DOX + CDDP for Reg-DI. Postoperative chemotherapy was intended to resume 3 weeks after surgery in both arms. The dataset consists of 497 consenting patients, prospectively enrolled between 1993 and 2002. More details about MRC BO06 can be found in the primary analysis of the trial [11].

\section{Sample selection}

From the original sample, we excluded 19 patients who did not start chemotherapy (13) or reported an abnormal dosage of one or both agents ( 6 , given dose $>1.25 \times$ prescribed dose). The outcome of interest is event-free survival (EFS), defined as time from the end of therapy until the occurrence of the first event defined as: local recurrence, evidence of new or progressive metastatic disease, second malignancy, death, or a combination of these events. We set a landmark at 180 days since registration, since nearly all patients enrolled in MRC BO06 completed the allocated regimen. For the analysis, we further excluded 57 patients, who reported an event within day 180 since randomization (50) or had not completed therapy by day 180 since randomization (7).

\section{Calculating target, achieved and regulated RDI}

Both target RDI (tRDI) and achieved RDI (aRDI) are obtained from the standardised cumulative dose $\delta$ and the standardised time on treatment $\tau$. The quantities $\delta$ and $\tau$ are computed on the prescribed regimen for tRDI, and on individual patients' treatment data for aRDI [19].

Regulated RDI (rRDI) extends the concept of aRDI by considering the longitudinal component: it is the evolution of the standardised cumulative dose $\delta$ over time. The new measure rRDI is a collection of values $(\delta, \tau)$ : each of these pairs corresponds to the cumulative dose and the cumulative time on treatment at the end of a completed cycle. More details about the computation of rRDI can be found in "Appendix 1".

\section{Displaying of treatment data in the time-dose plane}

Graphical techniques are used to visualise cumulative dose and treatment duration. We represent aRDI in the time-dose plane ( $\tau \delta$-plane), where the standardised time on treatment, $\tau$, is on the $x$-axis and the standardised cumulative dose received, $\delta$, on the $y$-axis. In the $\tau \delta$-plane, we represent patients' aRDI with points, while rRDI is represented by lines made of linear pieces. Each piece in the line 
corresponds to a cycle, where the slope represents the treatment intensity achieved in that period.

\section{Statistical methods}

We formed strata of patients according to tRDI, aRDI, and rRDI, and estimated EFS by applying Kaplan-Meier methodology on each stratification. We performed a landmark analysis at 180 days since randomization to investigate the association between RDI and EFS in the following scenarios: (i) patients are divided on the allocated regimen (Reg-C or Reg-DI) which leads to two strata of identical tRDI (tRDI-C, , tRDI-DI); (ii) patients are divided into four strata based on similarity of aRDI ( aRDI-0, aRDI-1, aRDI-2, aRDI-3); (iii) patients are divided into four strata based on similarity of rRDI ( rRDI-0, rRDI-1, rRDI-2, rRDI-3).

For scenarios (ii) and (iii), we formed post hoc patient groups (strata) by applying $k$-means clustering (unsupervised machine learning) of aRDI and rRDI separately. The choice of defining four strata was motivated in previous research on aRDI [19]. The $k$-means clustering methodology provides homogeneous strata with respect to aRDI or rRDI. Patients who reported a similar cumulative dose in a similar time window (aRDI) are grouped together; patients who reported a similar course of treatment (rRDI) are grouped together. Details are provided in "Appendix 2". For both aRDI and rRDI stratification, we also look at the distribution of tRDI across the strata and provide median values of $\tau, \delta$, and number of cycles.

All analyses are performed using Python 3.6.2 with pandas 0.20.2 [20], scikit-learn [21], and lifelines [22].

\section{Results}

Figure 1 shows patients in the $\tau \delta$-plane and the interrelationships between tRDI, aRDI, and rRDI. Figure 1a displays individual cumulative dose over time, i.e. rRDI lines, and compares them with those belonging to patients who reported no delays or reductions represented with the two thick black lines. Each line is obtained by joining the points $(\tau, \delta)$ corresponding to each cycle. In Fig. 1b, aRDI and tRDI can be compared with patients who reported no delays or reductions, represented in the figure as black square (Reg-C target) and dot (Reg-DI target). Figure 1c shows that rRDI extends aRDI over time, because the pattern of the final rRDI-value is identical to that of Fig. $1 b$.

Each rRDI line presents a sharp change in the steepness in the central part of Fig. 1 top and bottom right: this marks the last preoperative cycle whose duration includes the surgery window. rRDI lines form a tight bundle in the early phase of the treatment, but later they open up in a hand-fan shape because treatment adjustments are generally more frequent towards the end of the protocol.

Figure 2 shows the stratification based on aRDI; each colour represents a different post hoc strata. Strata are obtained at the landmark point-i.e. not at randomisation-by clustering patients with similar aRDI. Figure 3 shows rRDIbased stratification and individual rRDI lines for each group. Median values of relevant clinical quantities are reported in Table 1 for each group.

The groups obtained by clustering of aRDI/rRDI can be interpreted as follows.

\section{aRDI strata (different colours correspond to different strata)}

Stratum aRDI-0 is made of 34 patients of which 14 randomised to Reg-C (41\%) and 20 to Reg-DI (59\%). These are mostly patients who prematurely terminated the allocated protocol as the median cumulative dose received $(\delta)$ was $47 \%$ of the target one, while the median number of cycles completed is equal to 3 .

Strata aRDI-1 is made of 140 patients, of which 105 were randomised to Reg-C (75\%) and 35 to Reg-DI (25\%). The median cumulative dose received was $99 \%$ of the anticipated one, with a median of six cycles completed, and the median duration of the treatment was $1.56 \times 91=142$ days. This is interpreted as the subgroup of Reg-C patients who had expected complications during their course of treatment, anticipated in the clinical trial protocol, and it also contains patients of the Reg-DI group who reported unexpected complications and required strong treatment adaptations (cf. Figs. 1b, 2).

Stratum aRDI-2 is made of 210 patients, of which 39 were randomised to Reg-C (23\%) and 130 to Reg-DI (77\%). The median cumulative dose is $100 \%$ of the target one, and the median duration of their allocated treatment was $1.26 \times 91=115$ days. Patients of the DI-group in this stratum completed their allocated treatment whilst reporting expected adaptations, while patients of the C-group completed their allocated treatment following quite closely the anticipated schedule. It can be interpreted as the subgroup of patients randomised to Reg-DI who had a course of treatment with expected complications, but it also contains patients allocated to Reg-C who reported little to no complications.

Stratum aRDI-3 is made of 78 patients with 44 randomised to Reg-C (56\%) and 34 to Reg-DI (44\%). The median cumulative dose is $76 \%$ of the anticipated one, the median number of completed cycles is six, and the median duration of the treatment is $1.37 \times 91=125$ days. As the cumulative dose in this group is always larger than $50 \%$ and the treatment duration is generally larger than $1 \times 91=91$ days (cf. Fig. 2), we conclude that this group is formed by a 


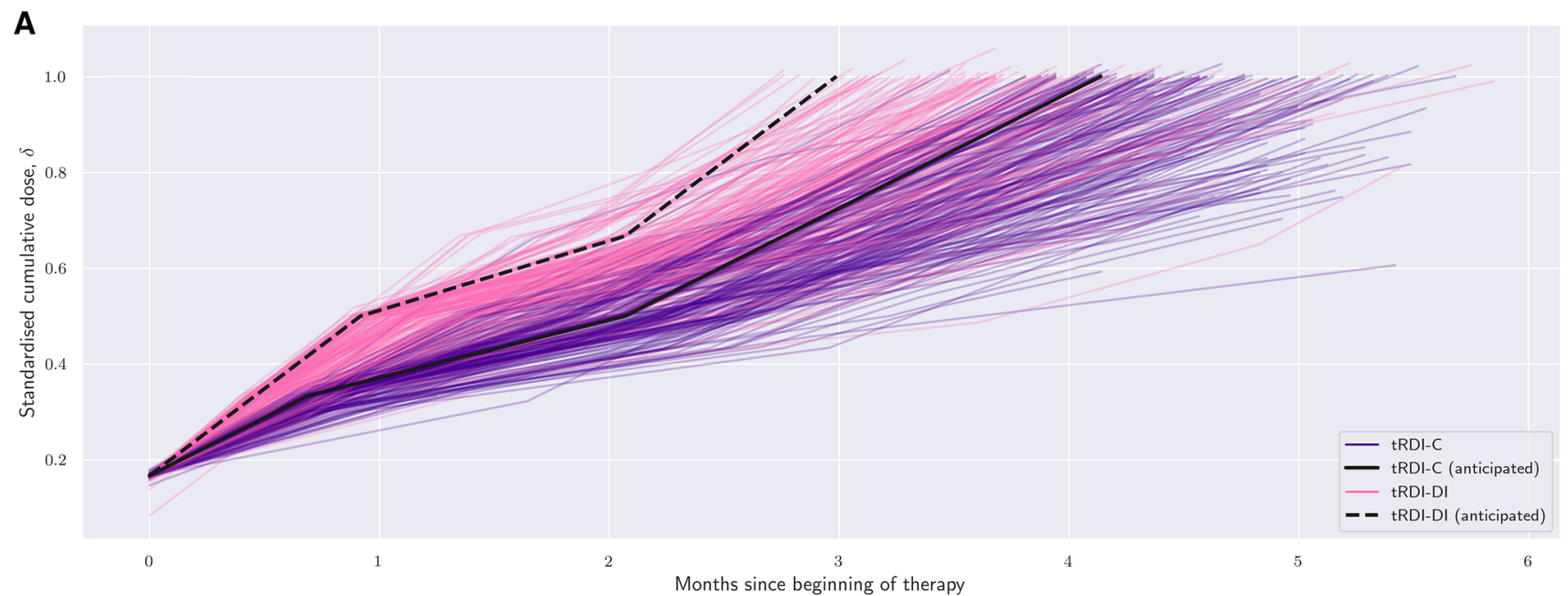

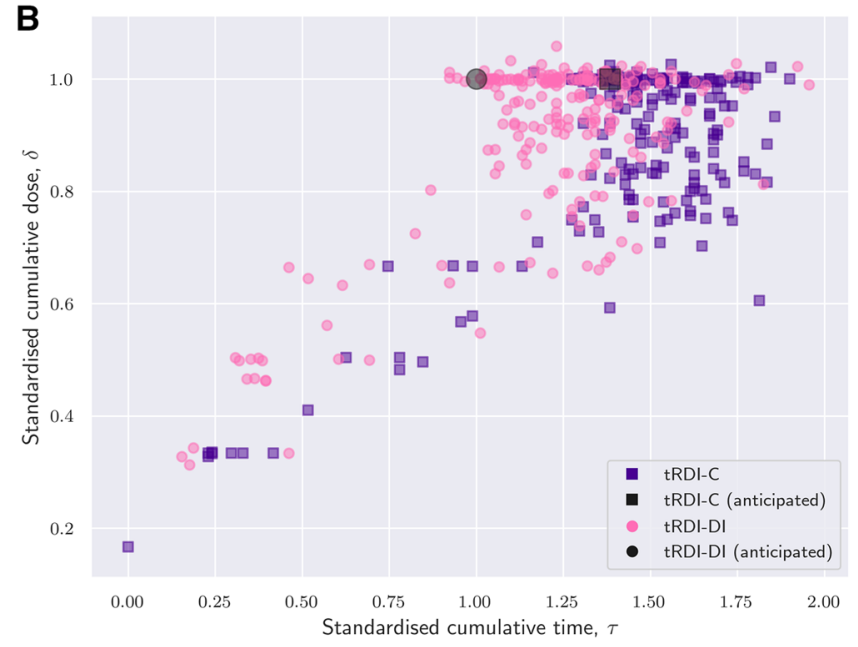

Fig. 1 a Individual standardised dose over time coloured by the allocated regimen (pink: Reg-DI; purple: Reg-C); each line is a graphical representation of the regulated RDI (rRDI) of a patient; black lines show the anticipated (target) rRDI line of a patient randomised to Reg-DI (dotted) or Reg-C (solid); divergence of coloured lines from the target black one illustrates how complicated an individual course of treatment was. b Individual cumulative standardised dose vs standardised treatment duration; each point is a graphical representation of the achieved RDI of a patient (pink circle: Reg-DI; purple square:

mixture of patients who terminated the allocated treatment in the postoperative phase or completed it reporting major reductions.

\section{rRDI strata (different colours correspond to different strata)}

Stratum rRDI-0 is made of 147 patients, of which 135 were randomised to Reg-C (92\%) and 12 to Reg-DI (8\%). The median cumulative dose is $97 \%$ of the target one, and the median duration of their allocated treatment was $1.48 \times 91=135$ days. This group contains $67 \%$ of all C-patients.

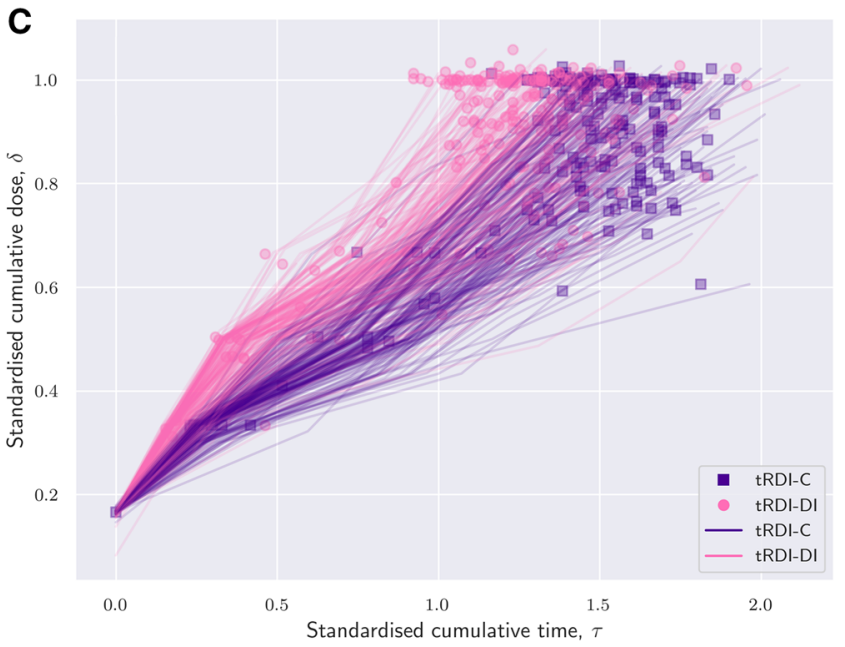

Reg-C); two thick black markers represent two fictitious patients who completed the protocol with no delays or dose reductions (dot: RegDI; square: Reg-C). These black markers are a graphical representation of target RDI. The larger the distance of a coloured point from the corresponding black one, the more complicated the individual course of treatment. With respect to $\mathbf{a}$, this view does not show where the complications were located in time. $\mathbf{c}$ As end points of rRDI lines match the pattern of achieved RDI visualised in $\mathbf{b}$, regulated RDI correctly extends achieved RDI over time

Stratum rRDI-1 is composed of 63 patients, of which 34 were randomised to Reg-C (54\%) and 29 to Reg-DI (46\%). The median cumulative dose was $58 \%$ of the target one, the median number of cycles complete was four, and the median time on treatment was $0.78 \times 91=71$ days. These are patients who discontinued the protocol or experienced some complications during the course of treatment.

Strata rRDI-2 is made of 92 patients randomised to RegDI, which accounts for $42 \%$ of all patients randomised to this regimen; it does not contain patients from the $\mathrm{C}$-group. The median cumulative dose was $100 \%$ of the target one, and the median duration of their allocated treatment was $1.19 \times 91=108$ days. 


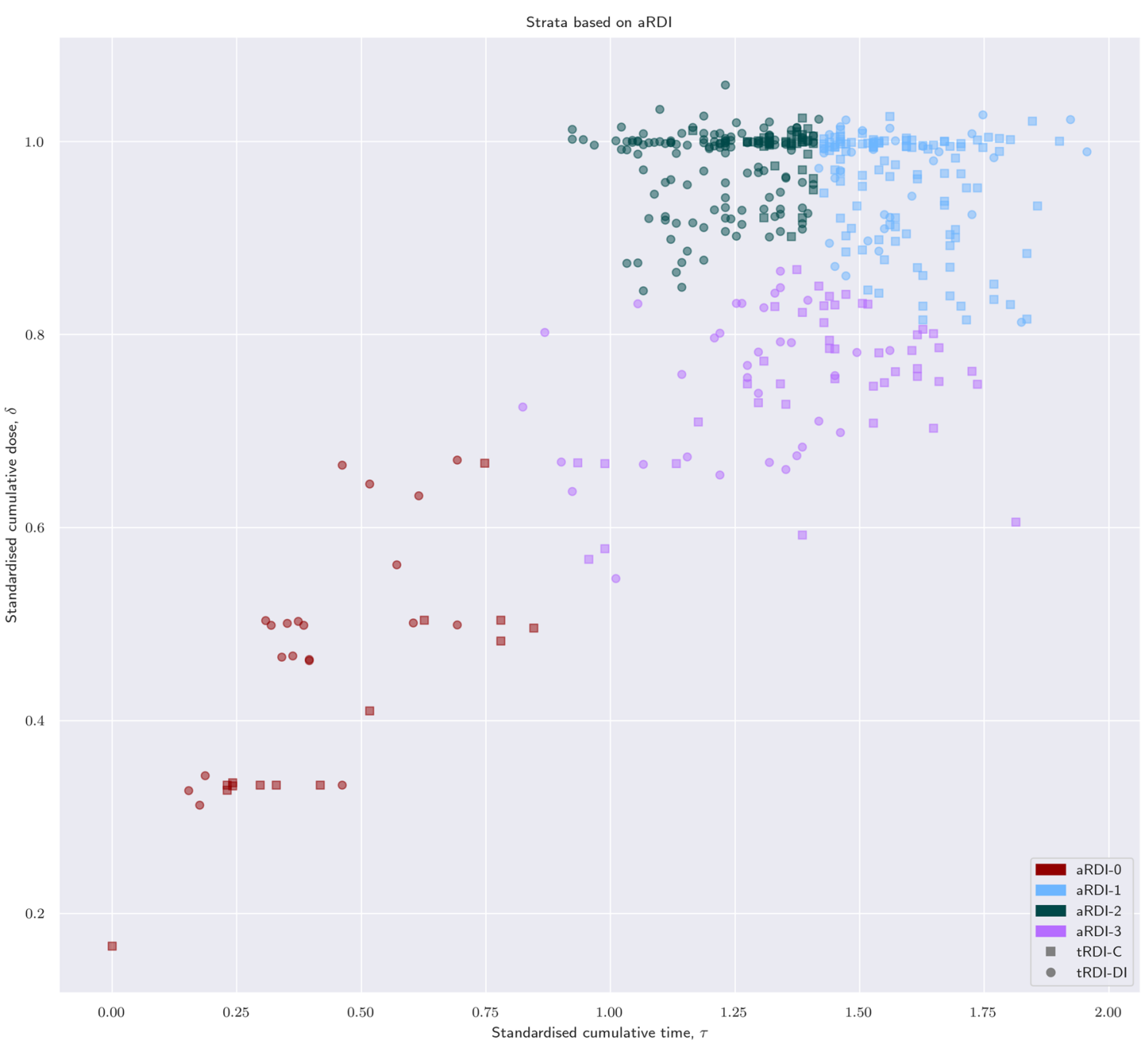

Fig. 2 Individual cumulative standardised dose versus standardised treatment duration; patients are coloured in four groups determined by similarity of achieved RDI; patients are marked according to their target RDI value (circle: patient randomised to Reg-DI; square: Reg-C)

It is interesting to compare this group to $r$ RD|-3: 129 patients, of which 33 were from the C-group (28\%) and 86 from the DI-group (72\%). The median cumulative dose was $94 \%$ of the target one, and the median duration of the protocol $1.40 \times 91=127$ days; $r$ RD|-3 contains $39 \%$ of all DI-patients enrolled to the trial. Figure 3 shows that, compared to rRDI-2, the rRDI-3 group shows less steep rRDI lines, i.e. cycle-wise less intense treatments. Since most patients were randomised to the same regimen (Reg-DI), the treatment-intensity reduction is due to a higher rate of toxicity-induced treatment adaptations in rRDI-3; see next section. Figure 3 also suggests that differences between rRDI-2 and rRDI-3 might be more evident in the preoperative phase.

\section{Adverse events in rRDI strata}

Figure 4 shows the proportion of patients in each rRDI stratum (relative to the group size) who required adaptations (either delays or dose reductions) according to the case report form. Each panel shows across cycles how many patients required treatment adaptations and the corresponding reason according to the case report form; cycles discontinued do not contribute to the plot. Groups rRDI- 0 and rRDI-2 (especially the latter) are driven by late myelotoxicity, but groups rRDI-1 and rRDI-3 by early myelotoxicity. In addition, rRDI-1 seems to be driven by 'other' reasons (most likely doxorubicin-induced cardiotoxicity, for which a dedicated checkbox was not present on the trial's case 


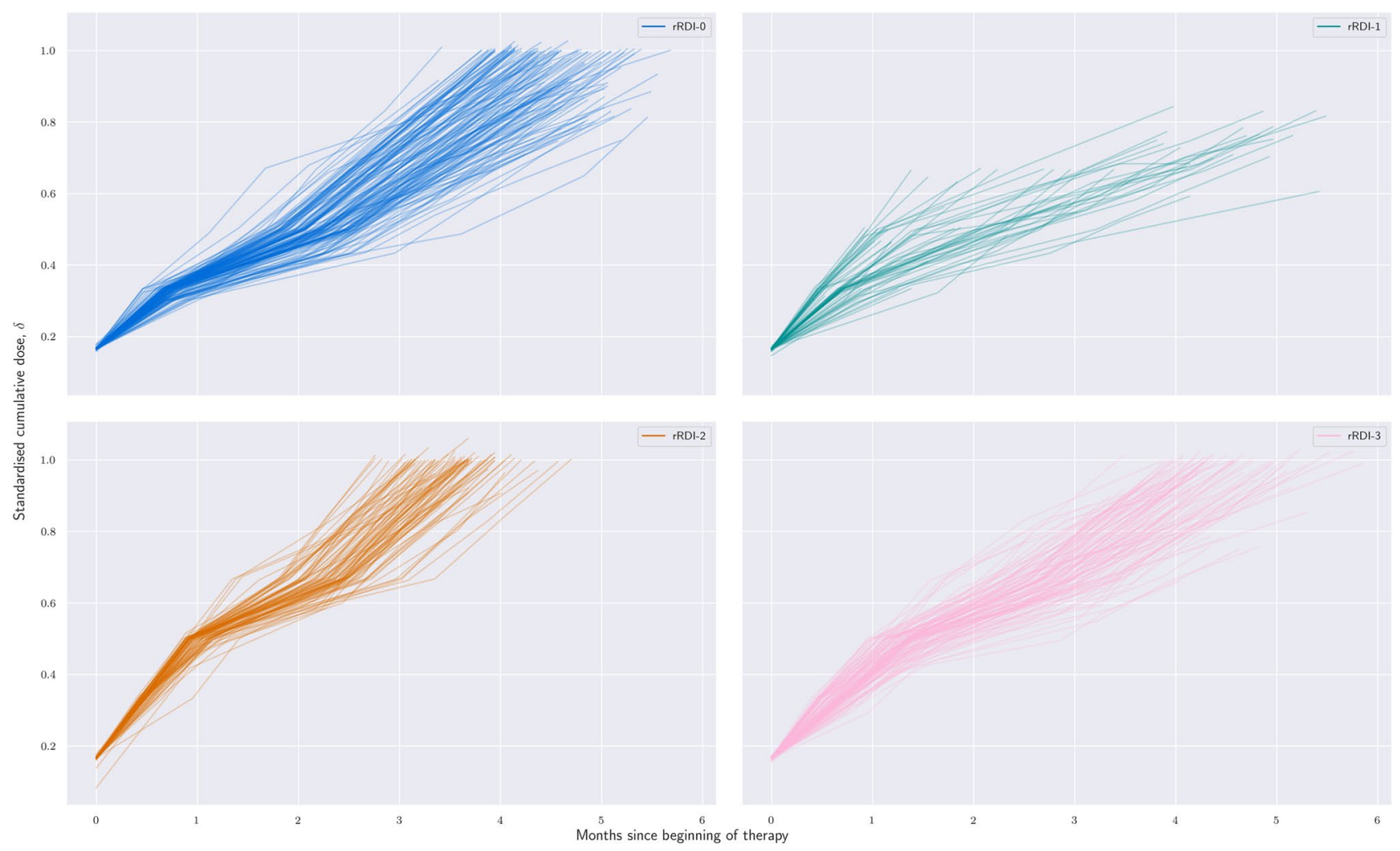

Fig. 3 Grouping of patients by similarity of regulated RDI and individual rRDI lines of each group; each line shows the growth of the standardised cumulative dose received over time. rRDI-0 is mostly composed of patients randomised to Reg-C (92\%), rRDI-1 captures treatment discontinuations and is balanced regimen-wise; rRDI-2 is composed only of patients allocated to Reg-DI; is composed for the large majority ( $72 \%$ ) by patients randomised to Reg-DI. rRDI-2 shows steeper lines than any other group, which means that patients therein reported the highest received dose intensity. In particular, $r$ RDI-2 presents a marked difference with rRDI-3 in terms of line steepness that is more evident in the preoperative part of the regimen

Table 1 Characteristics of strata obtained by clustering patients on similar aRDI and rRDI

\begin{tabular}{lllllllll}
\hline & N pts & \multicolumn{2}{l}{ N. Reg-C (\%) } & N. Reg-DI (\%) & \multicolumn{1}{l}{$\boldsymbol{\delta}$} & 工 & N. cycles \\
\hline aRDI-0 & 34 & 14 & $(41 \%)$ & 20 & $(59 \%)$ & 0.47 & 0.39 & 3 \\
aRDI-1 & 140 & 105 & $(75 \%)$ & 35 & $(25 \%)$ & 0.99 & 1.56 & 6 \\
aRDI-2 & 210 & 39 & $(23 \%)$ & 130 & $(77 \%)$ & 1.00 & 1.26 & 6 \\
aRDI-3 & 76 & 44 & $(56 \%)$ & 34 & $(44 \%)$ & 0.76 & 1.37 & 6 \\
\hline rRDI-0 & 147 & 135 & $(92 \%)$ & 12 & $(8 \%)$ & 0.97 & 1.48 & 6 \\
rRDI-1 & 63 & 34 & $(54 \%)$ & 29 & $(46 \%)$ & 0.58 & 0.78 & 4 \\
rRDI-2 & 92 & 0 & $(0 \%)$ & 92 & $(100 \%)$ & 1.00 & 1.19 & 6 \\
rRDI-3 & 129 & 33 & $(28 \%)$ & 86 & $(72 \%)$ & 0.94 & 1.40 & 6 \\
\hline
\end{tabular}

For each stratum, the table shows the total number of patients, percentage with respect to the allocated regimen, median values of standardised cumulative dose $(\delta)$, standardised cumulative time on treatment $(\tau)$, and number of cycles completed

report form); rRDI-3 reports the highest proportion of adjustment due to myelotoxicity in the preoperative period.

\section{Survival in strata of tRDI, aRDI, and rRDI}

Figure 5 shows KM estimation of EFS obtained by stratifying patients according to tRDI (Fig. 5a-1, a-2), aRDI (Fig. 5b1, b-2), or rRDI (Fig. 5c-1, c-2). For each estimated survival 

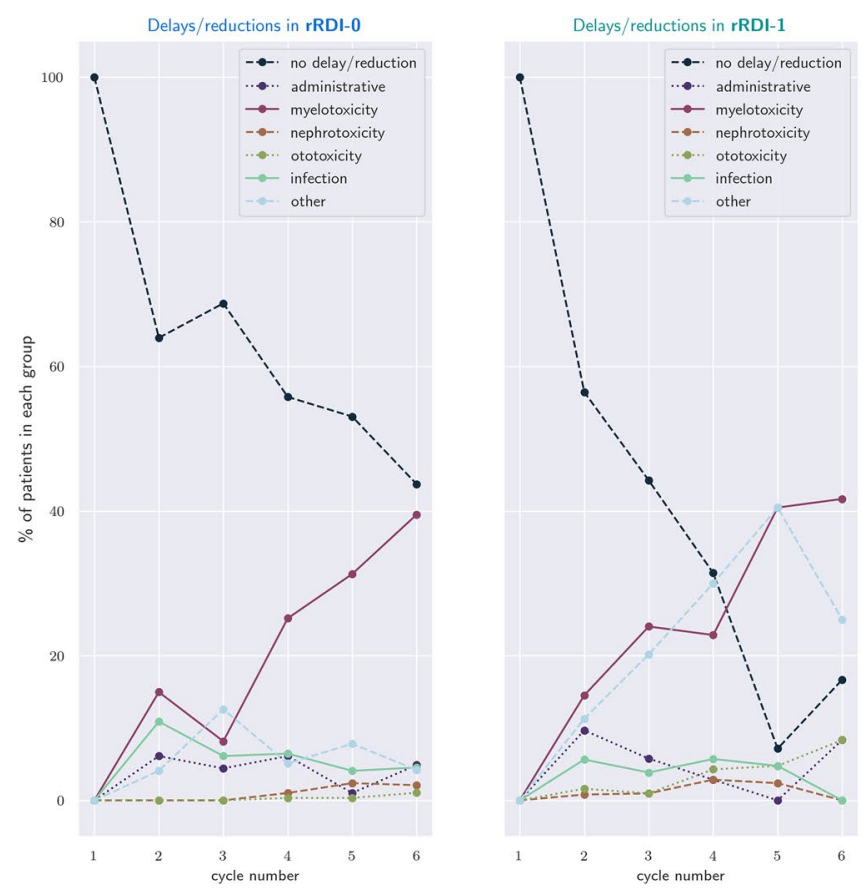

Fig. 4 Proportion of patient cycles that required adaptations (either delays or dose reductions) according to the study protocol and corresponding cause of adaptation as reported by the case report form. The view is per rRDI-group, i.e. per group of patients with similar regulated RDI pattern. The $x$-axis displays cycle number, while the $y$-axis shows the proportion of patients in the group who required adaptations. Groups rRDI-0 and rRDI-2 are driven by late mye-

curve, the corresponding patient stratification is shown on the left panel.

EFS for strata based on tRDI (Fig. 5a-2) are similar to the survival based on strata of aRDI (Fig. 5b-2). KM curves for tRDI-C, and tRDI-DI (Fig. 5a-2) are very similar to those of aRDI-1 and aRDI-2 (Fig. 5a-2), respectively. This is to be expected as these strata largely overlap pairwise.

However, stratifying patients according to rRDI (Fig. 5c) offers a very different picture. Patients in the group rRDI-1 show an EFS very similar to patients from the aRDI-0 group (Fig. 5c-2). Strata rRDI-0 and rRDI-2 show largely overlapping survival curves, with 3-year EFS smaller than 50\%. On the contrary, rRDI-3 (who had treatment-intensity reduction due to a high toxicity) shows better prognosis compared to strata rRDI-0 and rRDI-2, with 3-year EFS larger than $60 \%$. Strata are based only on individual treatment data; thus, the method is unaware of the efficacy outcome.

\section{Conclusions}

We presented a novel method to address the association between received dose intensity (RDI) and survival outcome by considering the treatment-intensity pattern achieved
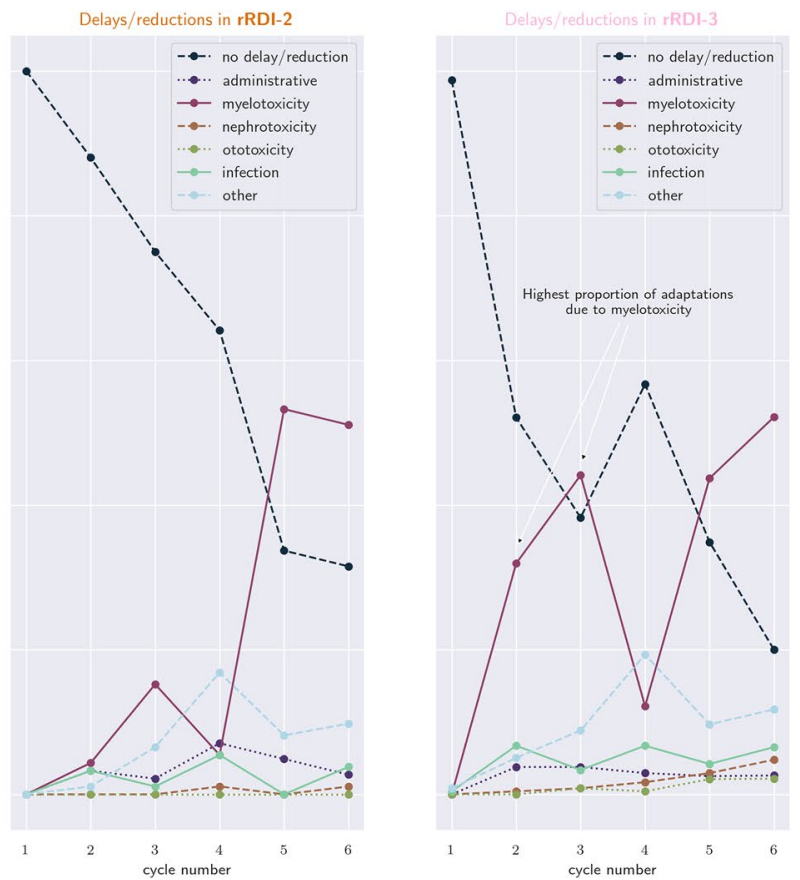

lotoxicity, while groups $\mathrm{rRDI}-1$ and $\mathrm{rRD} / \mathrm{-3}$ are driven by early myelotoxicity. In addition, rRDI-1 seems to be driven by 'other' reasons (most likely doxorubicin-induced cardiotoxicity, for which a dedicated checkbox was not present on the trial's case report form), and rRD|-3 reports the highest proportion of adjustment due to myelotoxicity in the preoperative period. Cycles discontinued do not contribute to the plot

by each patient. This approach offers new insight into the direction of personalised treatment, extending beyond osteosarcoma.

We investigated event-free survival in patient groups that are homogeneous with respect to RDI at a progressively more individualised level. This showed the benefits of investigating the association between RDI and survival at the patient level rather than at the usual cohort level.

The application of the method was shown using data from MRC BO06, a randomised clinical trial in osteosarcoma. This is an interesting dataset because many studies in osteosarcoma have challenged the benefits of escalating the dosage and/or compressing the regimen cohort-wide, showing complex interrelationships between increased RDI, histologic response, and outcome [23, 24].

In this manuscript, we offered a novel tool for tackling the problem at the patient level. While grouping patients according to the target dose intensity overlooks inter-patient differences, clustering based on the achieved intensity levels at the end of treatment does not consider which treatment adaptation patterns led to individual reductions and delays during the treatment. Conversely, grouping patients on similarity of received dosage patterns takes into account also the individual tolerability to the regimen. As a result, we highlighted 

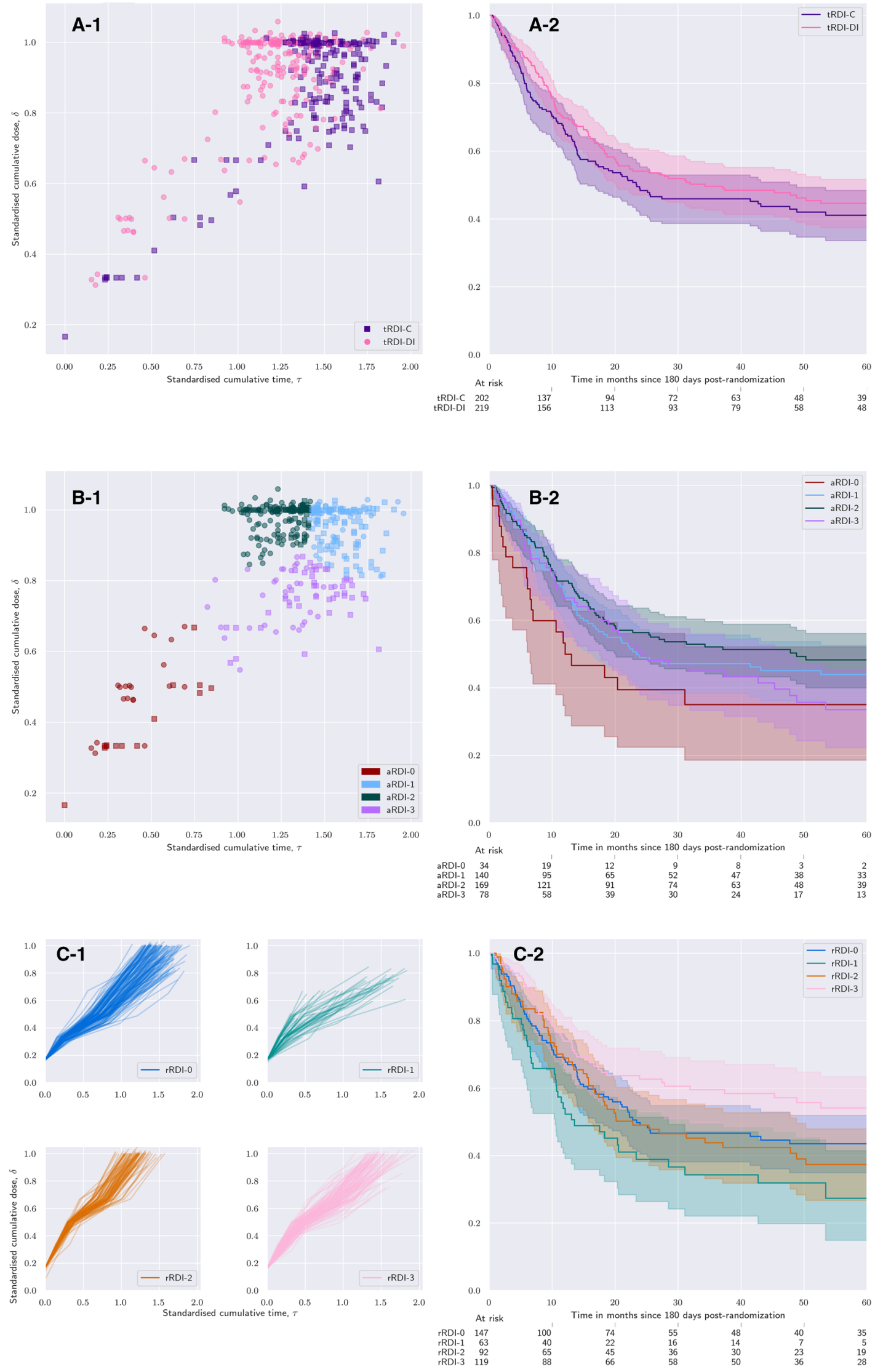
4Fig. 5 Estimation of event-free survival in groups that are homogeneous with respect to RDI using a progressively more individualised definition of RDI. a-1 Groups defined with respect to target RDI and a-2 the corresponding Kaplan-Meier curves; b-1 groups defined with respect to achieved RDI and $\mathbf{b}-\mathbf{2}$ the corresponding survival curves; c-1 groups defined with respect to regulated RDI and $\mathbf{c - 2}$ the corresponding survival curves

two subgroups of patients randomised to the dose-intense regimen who present quite different survival curves (rRDI-2 and rRDI-3). Remarkably, the subgroup with the worst survival ( $r R D \mid-2$, only patients from the DI-group and highest reported RDI) showed a KM curve that is nearly identical to the survival of the majority of patients from the C-group ( rRDI-0). This finding seems to be linked to the individual tolerability because rRDI-2 and rRDI-3 (both mostly formed by patients of the DI-group) present differences in the individual treatment intensity reported, especially in the preoperative cycles (Fig. 3). A comparison with Fig. 7 from [14] suggests that the potential implications of this fact might be osteosarcoma specific.

We have also shown that longitudinal treatment data contain information about the link between adverse events and survival outcome (cf. Figs. 4, 5c). As such, longitudinal treatment data might be used to link survival with drug metabolism when pharmacogenetics data are unavailable, like for MRC BO06. This link has been already investigated in small cell lung cancer through average binding occupancies [25], a measure of which is a well-known predictor of both outcome and skin disorders. Studies in metastatic colorectal cancer showed the effectiveness and safety of therapy schemes adapted on adverse events [26] or targeting toxicityrelated polymorphisms [27].

In conclusion, the strength of our method is the capability of detecting differences between patients randomised to the same regimen. Further, it seems to discriminate the prognostic value of chemotherapy-related complications over time. Indeed, rRDI-3 appears to be the subgroup of patients randomised to Reg-DI with the least tolerance to the regimen. In this respect, the manuscript offers a very precise view on the association between adverse events and osteosarcoma survival, which has been investigated through most severe chemotherapy-induced toxicity grades [28].

The method proposed here cannot be used yet in a prospective way. Yet, the results presented above suggest that there is room for gaining more insight into the effects of treatment intensification if longitudinal data and proper connected methodologies are used. On the one hand, our study calls for developing new methodologies targeted at cancer types where the link between treatment and survival is complex or confused. On the other, it calls for the collection of good-quality longitudinal data for both treatment and adverse events. Unfortunately, the level of detail available in the MRC BO06 dataset is not always accessible-an example is the recently concluded EURAMOS-1 trial. This in spite of treatment and adverse event data being longitudinal and already recorded per cycle in medical records. Clearly, making large datasets available with good-quality, longitudinal trial data might easily become an important issue for the organisation of future trials with respect to coordination efforts/overheads. This manuscript demonstrated that working in this direction might be a difficult, but rewarding approach.

Acknowledgements The authors thank Gordana Jovic and Medical Research Council for sharing the dataset used in this manuscript.

Funding This work was supported by the Dutch Foundation KiKa (Stichting Kinderen Kankervrij), Grant 163, through the project Metaanalysis of individual patient data to investigate dose-intensity relation with survival outcome for osteosarcoma patients.

\section{Compliance with ethical standards}

Conflict of interest The authors declare that they have no conflict of interest.

Research involving human participants and/or animals Permission to recruit patients to the MRC BO06 / EORTC 80931 protocol was provided by the appropriate national and local regulatory and local committees.

Informed consent Link-anonymised data were used for the purposes of this study, and the use of the data was consistent with the consent taken.

Availability of data and material The data that support the findings of this study are available from Medical Research Council, but restrictions apply to the availability of these data, which were used under license for the current study, and so are not publicly available.

OpenAccess This article is distributed under the terms of the Creative Commons Attribution 4.0 International License (http://creativeco mmons.org/licenses/by/4.0/), which permits unrestricted use, distribution, and reproduction in any medium, provided you give appropriate credit to the original author(s) and the source, provide a link to the Creative Commons license, and indicate if changes were made.

\section{Appendix 1: Definition of tRDI, aRDI, and rRDI}

Target received dose intensity (tRDI) is calculated as the ratio between standardised planned cumulative dose, $\delta$, and standardised planned duration of regimen, $\tau$. The former is obtained by standardising the sum of planned dose of all agents over all cycles of the allocated regimen; if a specific agent is not administered in a certain cycle, the contribution to the sum from that specific agent at that cycle is 0 . Standardisation is performed by dividing the cumulative planned dose by the cumulative planned dose of a reference regimen. In MRC BO06, 
the cumulative planned dose is the same for each arm, $6 \times(100+75)=1050 \mathrm{mg} / \mathrm{m}^{2}$, therefore standardisation with respect to Reg-DI yields $\delta=100 \%$ for both Reg-C and Reg-DI. The standardised planned duration of a regimen is obtained by standardising the difference in days between the planned start of the last and the first cycle of the regimen. Standardisation is performed by dividing the cumulative planned dose by the cumulative planned dose of a reference regimen. In MRC BO06, Reg-DI and Reg-C have planned duration of 91 and 126 days, respectively. Standardising with respect to Reg-DI yields a value of $\tau=91 / 126=72 \%$. The final computations of tRDI are as follows: for Reg-C, $1050 / 1050 \times 91 / 126=72 \%$; for RegDI, $1050 / 1050 \times 91 / 91=100 \%$.

Achieved received dose intensity (aRDI) is also calculated from standardised cumulative dose and standardised time-on-treatment, but these quantities are now computed on observed treatment data. For example, a patient reporting a $20 \%$ reduction in cycles 5 and 6 achieved a cumulative dose of $4 \times(100+75)+2 \times(100+75) \times 0.8=980 \mathrm{mg} / \mathrm{m}^{2}$, so $\delta=980 / 1050=93 \%$. In case an agent is omitted, the received dose of that agent in the formula is 0 for all the cycles affected by the omission; the same applies for all agents in case of discontinued cycles. Similarly, a patient who completed the protocol reporting a cumulative delay of 2 weeks (e.g. one 2-week delay or two 1-week delays at the beginning of two distinct cycles) would have $\tau=105 / 91=115 \%$ if allocated to Reg-DI, or $\tau=133 / 84=153 \%$ if allocated to Reg-C. In general, two patients-even two Reg-C or two Reg-DI-will report different values of $\tau$ and $\delta$ depending on the individual realisation of their intended treatment, i.e. depending on the delays and dose reductions reported throughout the treatment. In the graphical representation, the ratio $\delta / \tau$ is the slope of a line connecting the patient point with the origin $(0,0)$ of the $\tau \delta$-plane. For patients who completed all planned cycles, the quantity $\delta / \tau$ is close to the aRDI as defined in [14]. For patients who did not complete all planned cycles, the ratio $\delta / \tau$ might be difficult to use in practice. A solution is either to work with the pair $(\delta, \tau)$ or to multiply $\delta / \tau$ by the proportion of cycles completed.

Regulated received dose intensity (rRDI) is the evolution of the standardised cumulative dose over time; rRDI is a function of the time on treatment: for each time $t$, rRDI is the aRDI that patients would report in case of protocol discontinuation at exactly that moment. Both regimens of MRC BO06 were composed of six cycles, where both agents were administered at the beginning of each cycle. Thus, the standardised cumulative dose will increase at most six times during the administration of the regimen (it will increase less times only in case of discontinuation). This means that for MRC BO06, rRDI can be represented as a sequence of (at most) six values, i.e. the sequence of aRDI values that patients would report if they discontinued after each cycle was administered.

For a fictitious DI-patient, who completed the protocol without delays or dose reductions, rRDI can be displayed as the sequence of $(\delta, \tau)$-values $[(0.167,0 / 91),(0.333$, 14/91), (0.500, 28/91), (0.667, 63/91), (0.833, 77/91), $(1.000,91 / 91)]$; for the corresponding C-patient, [ $(0.167$, 0/91), (0.333, 21/91), (0.500, 63/91), (0.667, 84/91), (0.833, $105 / 91),(1.000,126 / 91)]$.

\section{Appendix 2: Clustering of patients based on achieved and regulated received dose intensity (aRDI and rRDI)}

Clustering forms groups of patients such that members of the same group are similar with respect to received dose intensity.

Clustering based on achieved received dose intensity (aRDI) is performed by grouping patients with similar values of $\delta$ and $\tau$. In other words, clustering returns groups of patients who were administered a similar cumulative dose in a similar time window. This means that patients assigned to the same cluster will report similar aRDI, or closer to aRDI of an average patient in the same group than to aRDI of average patient from other groups. In particular, this kind of clustering will not separate well Reg-DI and Reg-C patients. Instead, it will group Reg-DI patients with large cumulative delays (due to a problematic course of therapy) with Reg-C with a regular course of therapy; it will also tend to group Reg-DI patients with a regular course of therapy with Reg-C patients who reported less than average or no delays at all.

Clustering based on regulated received dose intensity (rRDI) is performed by grouping patients with a similar course of therapy. Groups are formed using the relative increase of the cumulative dose over time. As explained in "Appendix 1", rRDI can be represented as a sequence of $(\delta, \tau)$-values, one pair for each cycle administered. In case of MRC BO06, there can be up to $6(\delta, \tau)$-pairs. In other words, rRDI of a MRC BO06 patients can be described by the sequence $\left[\left(\delta_{1}, \tau_{1}\right),\left(\delta_{2}\right.\right.$, $\left.\left.\tau_{2}\right), \ldots,\left(\delta_{6}, \tau_{6}\right)\right]$, where $\delta_{i}$ and $\tau_{i}$ are the standardised cumulative dose and time on treatment up to at the beginning of the $i$ th cycle. The slopes are obtained as $\rho_{\mathrm{i}}=\left(\delta_{i+1}-\delta_{i}\right) /\left(\tau_{i+1}-\tau_{i}\right)$, $i=1,2, \ldots, 5$. The slopes measure the intensity of the treatment between the $i$ th and the $(i+1)$ th cycle: if no reduction was applied to the nominal dosage of cycle $i+1$, the increase $\left(\delta_{i+1}-\delta_{i}\right)$ equals $1 / 6$ for both a Reg-DI and a Reg-C patient; if the start of cycle $i+1$ was not delayed, the increase $\left(\tau_{i+1}-\tau_{i}\right)$ equals $14 / 84$ for a Reg-DI patient and 21/84 for a Reg-C patient. As a consequence, $\rho_{i}$ equals, respectively, 1 or $2 / 3$ for a Reg-DI and a Reg-C patient who did not report delays or dose reductions in cycle $i+1$. If a delay or a reduction is applied, then $\rho_{i}$ is smaller. In case a patient completes less 
cycles than anticipated, some $\rho_{i}$ cannot be calculated and are replaced by 0 . Clustering is performed by grouping patients based on the similarity of the whole sequence of values $\left[\rho_{1}\right.$, $\left.\rho_{2,} \rho_{3,} \rho_{4}, \rho_{5}\right]$. With this method, groups tend to separate better Reg-C and Reg-DI patients, because information on the intended treatment (duration of cycles) is embedded in $\rho_{i}$ at the cycle level and not just regimen-wise. Moreover, $\rho_{i}$ carries information about the medical interventions that occurred in each cycle, offering a surrogate measure of individual tolerability to the allocated regimen.

\section{References}

1. Hryniuk WM, Goodyear M (1990) The calculation of received dose intensity. J Clin Oncol 8(12):1935-1937

2. Hryniuk WM, Frei E 3rd, Wright FA (1998) A single scale for comparing dose-intensity of all chemotherapy regimens in breast cancer: summation dose-intensity. J Clin Oncol 16(9):3137-3147

3. Citron ML (2004) Dose density in adjuvant chemotherapy for breast cancer. Cancer Investig 22(4):555-568

4. Wildiers H, Reiser M (2001) Relative dose intensity of chemotherapy and its impact on outcomes in patients with early breast cancer or aggressive lymphoma. Crit Rev Oncol Hematol 77(3):221-240

5. Yuan JQ, Wang SM, Tang LL, Mao J, Wu YH, Hai J et al (2015) Relative dose intensity and therapy efficacy in different breast cancer molecular subtypes: a retrospective study of early stage breast cancer patients treated with neoadjuvant chemotherapy. Breast Cancer Res Treat 151(2):405-413

6. Shirotake S, Yasumizu Y, Ito K, Masunaga A, Ito Y, Miyazaki Y et al (2016) Impact of second-line targeted therapy dose intensity on patients with metastatic renal cell carcinoma. Clin Genitourin Cancer 14(6):e575-e583

7. Yabusaki N, Fujii T, Yamada S, Murotani K, Sugimoto H, Kanda $\mathrm{M}$ et al. The significance of relative dose intensity in adjuvant chemotherapy of pancreatic ductal adenocarcinoma-including the analysis of clinicopathological factors influencing relative dose intensity. Medicine 2016;95(29):e4282

8. Loschi S, Dufour C, Oberlin O, Goma G, Valteau-Couanet D, Gaspar N (2015) Tandem high-dose chemotherapy strategy as first-line treatment of primary disseminated multifocal Ewing sarcomas in children, adolescents and young adults. Bone Marrow Transplant 50(8):1083-1088

9. Ozols RF (2007) Ovarian cancer: is dose intensity dead? J Clin Oncol 25(27):4157-4158

10. Livingston RB (1994) Dose intensity and high dose therapy: two different concepts. Cancer 74(S3):1177-1183

11. Lewis IJ, Nooij MA, Whelan J, Sydes MR, Grimer R, Hogendoorn $P C$ et al (2007) Improvement in histologic response but not survival in osteosarcoma patients treated with intensified chemotherapy: a randomized phase III trial of the European Osteosarcoma Intergroup. J Natl Cancer Inst 99(2):112-128

12. Marina N, Bielack S, Whelan J, Smeland S, Krailo M, Sydes MR et al (2009) International collaboration is feasible in trials for rare conditions: the EURAMOS experience. In: Jaffe N, Bruland OS, Bielack S (eds) Pediatric and adolescent osteosarcoma. Springer, Boston, pp 339-353

13. Marina NM, Smeland S, Bielack SS, Bernstein M, Jovic G, Krailo MD et al (2016) Comparison of MAPIE versus MAP in patients with a poor response to preoperative chemotherapy for newly diagnosed high-grade osteosarcoma (EURAMOS-1): an openlabel, international, randomised controlled trial. Lancet Oncol 17(10):1396-1408
14. Lewis IJ, Weeden S, Machin D, Stark D, Craft AW, European Osteosarcoma Intergroup (2000) Received dose and dose-intensity of chemotherapy and outcome in nonmetastatic extremity osteosarcoma. J Clin Oncol 18(24):4028-4037

15. Collins M, Wilhelm M, Conyers R, Herschtal A, Whelan J, Bielack S et al (2013) Benefits and adverse events in younger versus older patients receiving neoadjuvant chemotherapy for osteosarcoma: findings from a meta-analysis. J Clin Oncol 31(18):2303-2312

16. Windsor RE, Strauss SJ, Kallis C, Wood NE, Whelan JS (2012) Germline genetic polymorphisms may influence chemotherapy response and disease outcome in osteosarcoma. Cancer 118(7):1856-1867

17. Hagleitner MM, Coenen MJ, Gelderblom H, Makkinje RR, Vos HI, de Bont ES et al (2015) A first step toward personalized medicine in osteosarcoma: pharmacogenetics as predictive marker of outcome after chemotherapy-based treatment. Clin Cancer Res 21(15):3436-3441

18. Ray-Coquard I, Cropet C, Van Glabbeke M, Sebban C, Le Cesne A, Judson I et al (2009) Lymphopenia as a prognostic factor for overall survival in advanced carcinomas, sarcomas, and lymphomas. Cancer Res 69(13):5383-5391

19. Lancia C, Anninga J, Spitoni C, Sydes MR, Whelan J, Hogendoorn PCW, Gelderblom AJ, Fiocco M (2018) A method to measure the mismatch between target and achieved received dose intensity of chemotherapy in cancer studies: the application to osteosarcoma randomised trial MRC-BO06. BMJ Open (Accepted)

20. McKinney W. Data structures for statistical computing in Python. In: Proceedings of the 9th Python in science conference; 2010 Jun 28, Austin, TX, pp 51-56

21. Pedregosa F, Varoquaux G, Gramfort A, Michel V, Thirion B, Grisel O et al (2011) Scikit-learn: machine learning in Python. J Mach Learn Res 12(Oct):2825-2830

22. Davidson-Pilon C, Kalderstam J, Kuhn B, Fiore-Gartland A, Parij A, Stark K et al (2018) Lifelines. version 0.11.1. https://githu b.com/CamDavidsonPilon/lifelines/. Accessed 1 Feb 2019

23. Luetke A, Meyers PA, Lewis I, Juergens H (2014) Osteosarcoma treatment-where do we stand? A state of the art review. Cancer Treat Rev 40(4):523-532

24. Ta HT, Dass CR, Choong PF, Dunstan DE (2009) Osteosarcoma treatment: state of the art. Cancer Metastasis Rev 28:247-263

25. Kimura K, Takayanagi R, Fukushima T, Yamada Y (2017) Theoretical method for evaluation of therapeutic effects and adverse effects of epidermal growth factor receptor tyrosine kinase inhibitors in clinical treatment. Med Oncol 34(10): 178

26. Van Cutsem E, Tejpar S, Vanbeckevoort D, Peeters M, Humblet Y, Gelderblom H et al (2012) Intrapatient cetuximab dose escalation in metastatic colorectal cancer according to the grade of early skin reactions: the randomized EVEREST study. J Clin Oncol 30(23):2861-2868

27. Kanekiyo S, Hazama S, Kondo H, Nagashima A, Eto R, Yoshida $S$ et al (2013) UDP-glucuronosyltransferase (UGT) $1 \mathrm{~A} 1 * 28$ polymorphism-directed phase II study of irinotecan with 5'-deoxy5 -fluorouridine (5'-DFUR) for metastatic colorectal cancer. Anticancer Res 33(8):3423-3430

28. McTiernan A, Jinks RC, Sydes MR, Uscinska B, Hook JM, van Glabbeke $M$ et al (2012) Presence of chemotherapy-induced toxicity predicts improved survival in patients with localised extremity osteosarcoma treated with doxorubicin and cisplatin: a report from the European Osteosarcoma Intergroup. Eur J Cancer 48(5):703-712

Publisher's Note Springer Nature remains neutral with regard to jurisdictional claims in published maps and institutional affiliations. 


\section{Affiliations}

\section{Carlo Lancia $^{1}$ (D) Jakob K. Anninga ${ }^{2}$ - Matthew R. Sydes ${ }^{3}$ (D) Cristian Spitoni $^{4,5}$ - Jeremy Whelan ${ }^{6}$ (D) . Pancras C. W. Hogendoorn ${ }^{7}$ (D) Hans Gelderblom ${ }^{8}$ (D) Marta Fiocco ${ }^{1,9}$}

Jakob K. Anninga

jakob.anninga@ radboudumc.nl

Matthew R. Sydes

m.sydes@ucl.ac.uk

Jeremy Whelan

jeremy.whelan@uclh.nhs.uk

Pancras C. W. Hogendoorn

p.c.w.hogendoorn@lumc.nl

Hans Gelderblom

a.j.gelderblom@lumc.nl

Marta Fiocco

m.fiocco@lumc.nl

1 Mathematical Institute Leiden University, Niels Bohrweg 1, 2333 CA Leiden, The Netherlands

2 Department Paediatric Oncology, Radboud University Medical Center, Geert Grooteplein Zuid 10, 6525 GA Nijmegen, The Netherlands
3 MRC Clinical Trials Unit at UCL, Institute of Clinical Trials and Methodology, UCL and MRC London Hub for Trials Methodology Research, 90 High Holborn, London WC1V 6LJ, UK

4 Mathematical Institute Utrecht University, Budapestlaan 6, 3584 CD Utrecht, The Netherlands

5 Department of Epidemiology, University Medical Center Utrecht, Heidelberglaan 100, 3584 CX Utrecht, The Netherlands

6 Department of Oncology, University College London Hospital, 235 Euston Rd, Fitzrovia, London NW1 2BU, UK

7 Department of Pathology, Leiden University Medical Center, Albinusdreef 2, 2333 ZA Leiden, The Netherlands

8 Department of Medical Oncology, Leiden University Medical Center, Albinusdreef 2, 2333 ZA Leiden, The Netherlands

9 Department of Biomedical Data Science, Section Medical Statistics, Leiden University Medical Center, Albinusdreef 2, 2333 ZA Leiden, The Netherlands 